\title{
Leptomeningeal Rheumatoid Nodules: Diagnosis and Challenging Therapeutics
}

\author{
Cassie Nesbitt, ${ }^{1,}$ Luke Wilshire, Doreen Quan, ${ }^{2}$ Cameron Shaw, ${ }^{3}$ and Peter Batchelor ${ }^{3}$ \\ ${ }_{1}^{1}$ Department of Neurology, Barwon Health/Geelong University Hospital, Geelong, Australia \\ 2 Department of Radiology, Barwon Health/Geelong University Hospital, Geelong, Australia \\ 3 Department of Neuroscience, Barwon Health/Geelong University Hospital, Geelong, Australia \\ *Corresponding author:Cassie Nesbitt, Department of Neurology, Barwon Health/Geelong University Hospital, P. O. Box: 281, Geelong, Australia. Tel:+61-404208994, Fax:+3-42150757, \\ E-mail:cassie_nesbitt@hotmail.com
}

Received: January 12, 2015; Accepted: February 24, 2015

\begin{abstract}
Introduction: Leptomeningeal rheumatoid nodules are a rare condition. This case demonstrates the unique presentation and diagnostic workup for the distinctive condition and highlights the difficulties surrounding therapeutics.

Case Presentation:A 67 year old woman presented with recurrent transient ischaemic attack-like episodes over a 2 year period. Nodular enhancing leptomeningeal changes were detected on MRI and consistent with meningeal rheumatoid nodules on biopsy. The patient's nodular disease continued to progress and regress clinically and radiologically irrespective of disease modifying agents and peripheral and serological rheumatoid arthritis control.

Conclusions:This patient's unique presentation and diagnostic work-up is discussed alongside the dilemma of therapeutic management of meningeal rheumatoid nodules.
\end{abstract}

Keywords: Leptomeningeal; Rheumatoid; Nodules; Pachymeningitis; Tocilizumab; Brain; Biopsy

\section{Introduction}

Inflammatory central nervous system (CNS) disease with associated leptomeningeal rheumatoid nodules is a rare complication of rheumatoid arthritis that is diagnostically and therapeutically challenging. No current therapies are known to effect regression or long term control of CNS nodular disease. Monitoring response to treatment can also be problematic as radiological changes evolve slowly and patients often lack clinical deficits. This case illustrates the distinctive presentation and diagnostic work-up of this condition along with the therapeutic difficulties.

\section{Case Presentation}

A 67 year old woman presented to our Neurology Clinic with a 2 week history of motor and sensory symptoms affecting the right side of her face and right lower limb, giving an impression of recurrent transient ischaemic attacks (TIA). Each episode lasted between 10 and 20 minutes followed by complete resolution.

Her only past medical history was of rheumatoid arthritis, well controlled with weekly doses of methotrexate and leflunomide. The patient had no deficits at the time of presentation and her neurological examination was unremarkable. She denied constitutional or infective symptoms.

\subsection{Investigations}

A brain MRI demonstrated abnormal signal in the frontal cortex bilaterally with associated hyperintensity on the fluid-attenuated inversion recovery (FLAIR) images. These findings were interpreted as vascular engorgement with post-contrast enhancement of the leptomeninges Figure 1. Several differential diagnoses were considered including malignancy, vasculitis, reversible cerebral vasoconstriction syndrome and other leptomeningeal pathologies.

The patient underwent follow-up MRI at 2 months which demonstrated progression of the leptomeningeal enhancement. Analysis of cerebrospinal fluid (CSF) revealed mildly elevated protein of $0.78 \mathrm{~g} / \mathrm{L}(0.12-0.6 \mathrm{~g} / \mathrm{L})$ and a pleocytosis of white blood cells $\left(5 \times 10^{6} / \mathrm{L}\right)$ and red blood cells $(42$ $\times 10^{6} / \mathrm{L}$ ). The CSF angiotensin converting enzyme (ACE) was negative and cytology demonstrated no malignant cells.

The possibility of malignancy was further investigated with a CT scan of the chest, abdomen and pelvis which demonstrated two left upper lobe lung nodules; both unremarkable on whole body PET scan. The PET scan did reveal a solitary left thyroid hypermetabolic nodule, proven to be benign on fine needle aspirate.

Serum vasculitis screening revealed a rheumatoid factor of $109 \mathrm{kIU} / \mathrm{L}$, double stranded DNA of $4.4 \mathrm{IU} / \mathrm{mL}(<$ 4.2 ) and an ANA (anti nuclear factor) titre of $40(<160)$.

Copyright ( 2015, Tehran University of Medical Sciences. This is an open-access article distributed under the terms of the Creative Commons Attribution-NonCommercial 4.0 International License (http://creativecommons.org/licenses/by-nc/4.0/) which permits copy and redistribute the material just in noncommercial usages, provided the original work is properly cited. 
Her inflammatory markers included an ESR (erythrocyte sedimentation rate) of $62 \mathrm{~mm} /$ hour ( $0-20 \mathrm{~mm} /$ hour) and CRP (C reactive protein) of $10 \mathrm{mg} / \mathrm{L}(<2.9 \mathrm{mg} / \mathrm{L})$.

Because of the persistent leptomeningeal enhancement of uncertain aetiology, a meningeal biopsy was performed. Histological findings were consistent with a diagnosis of meningeal rheumatoid nodules with giant cells, necrotising granulomas and palisaded histiocytes Figure 2.

\subsection{Differential Diagnosis}

Various conditions can mimic the clinical presentation or the MRI enhancement pattern seen in our patient's initial presentation, including leptomenin- geal metastases and metastatic disease in locations adjacent to the nervous system, infectious and autoimmune disease affecting the leptomeninges and/or nerve roots, late effects of prior treatments such as radiotherapy, post lumbar puncture changes and other imaging artefacts.

Leptomeningeal metastasis is one of the common causes of meningeal thickening and enhancement and the patient was extensively investigated to exclude underlying malignancy. Although the patient was immunosuppressed, her condition was not sufficiently severe or acute to consider diagnoses such as acute bacterial meningitis. Moreover, CSF gram stain, cytology and ACE studies were all negative.

Figure 1. Initial MRI Study (Transverse Flair and Coronal T1)
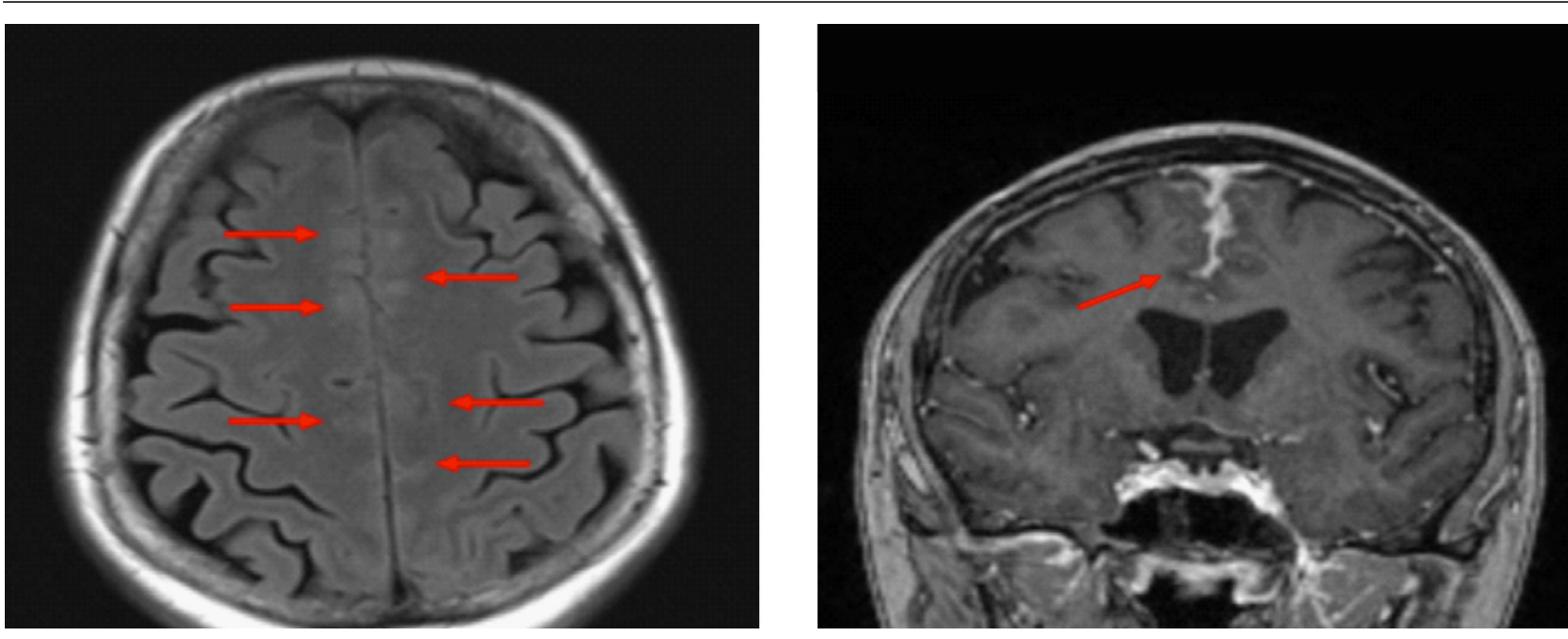

Abnormal signal in the frontal cortex bilaterally with associated hyperintensity on FLAIR images interpreted as vascular engorgement with post-contrast enhancement and thickening of the leptomeninges.

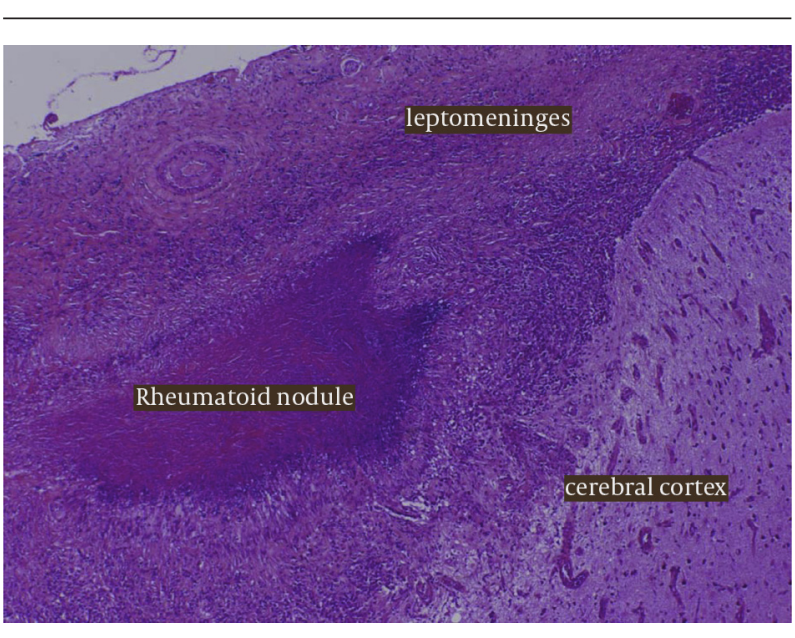

Figure 2. Histology From The Meningeal Biopsy Highlighting The Presence of Giant Cells, Necrotising Granulomas and Palisaded Histiocytes All Consistent With an Underlying an Inflammatory Pach-Meningitis and Leptomeningeal Rheumatoid Nodules
Inflammatory causes such as ANCA-positive vasculitis and systemic lupus erythematosus were excluded with a vasculitic screen, and her rheumatoid factor and inflammatory markers were in keeping with known rheumatoid disease. Given the clinical context, we progressed to meningeal biopsy as a definitive means of diagnosing leptomeningeal rheumatoid nodules.

Idiopathic pachymeningitis has recently been associated with immunoglobulin (Ig) G-4 related disease, and this new condition has also been linked with rheumatoid disease (1). However, this diagnosis was felt to be unlikely, with investigations demonstrating normal serum IgG and subclass levels. In addition, the meningeal biopsy showed an IgG to IgG4 ratio of $15 \%$, of less than $40 \%$ are thought to exclude IgG-4 related disease.

\subsection{Treatment}

The patient had well-controlled rheumatoid arthritis at the time of her presentation, with no swollen joints and only minor episodes of synovitis. She had two pe- 
ripheral rheumatoid nodules on her right hand with no functional limitation. She was receiving immunosuppressive therapy with methotrexate and leflunomide.

The patient was initially commenced on aspirin and a calcium channel blocker and was treated empirically with colchicine therapy ( $0.5 \mathrm{mg}$ twice daily) in an attempt to reduce the nodular disease. Imaging performed 3 months after commencing colchicine demonstrated stability of the leptomeningeal thickening and a reduction in the hyperintensity surrounding the frontal cortex on T2-weighted and FLAIR studies.

The patient continued to undergo surveillance MRI every 3 months along with neurological and rheumatological reviews. She remained asymptomatic for a 2 year period and MRI studies revealed regression of the initial changes involving the bilateral frontal cortex. Nevertheless, subsequent progression was evident in other areas particularly the left parietal lobe which demonstrated subtle diffusion restriction Figure 3.

The imaging changes suggested disease progression in the face of disease modifying immunosuppressants and raised the dilemma of whether to pursue more aggressive disease modifying agents or adopt a circumspect management plan given the patient's asymptomatic status. No clinical trial evidence was available to support the effectiveness of an aggressive treatment approach. The Methotrexate was weaned to establish whether the agent was perpetuating rheumatoid nodule formation.

\subsection{Outcome and Follow-Up}

The patient experienced further TIA-like symptoms 2 years after her initial presentation, with acute on subacute dysphasia which subsided over several days with no specific treatment. MRI excluded ischaemic stroke and revealed a progression of patchy leptomeningeal thickening with enhancement particularly over left frontal lobe as well as some regression in the parietal areas.

The imaging also revealed widespread areas of punctuate leptomeningeal diffusion restriction. This was suggestive of either a microvascular obliterative process or mild hydrocephalus, presumably related to a reduced CSF resorptive capacity.

The patient was then commenced on a trial of Tocilizumab (a humanized monoclonal antibody against the interleukin [IL] 6 receptor) with leflunomide also being ceased. The patient has currently been on Tocilizumab for 18 months and is asymptomatic with surveillance MRI revealing ongoing regression and no further progression. We feel it is still too early to fully determine the success of Tocilizumab therapy.
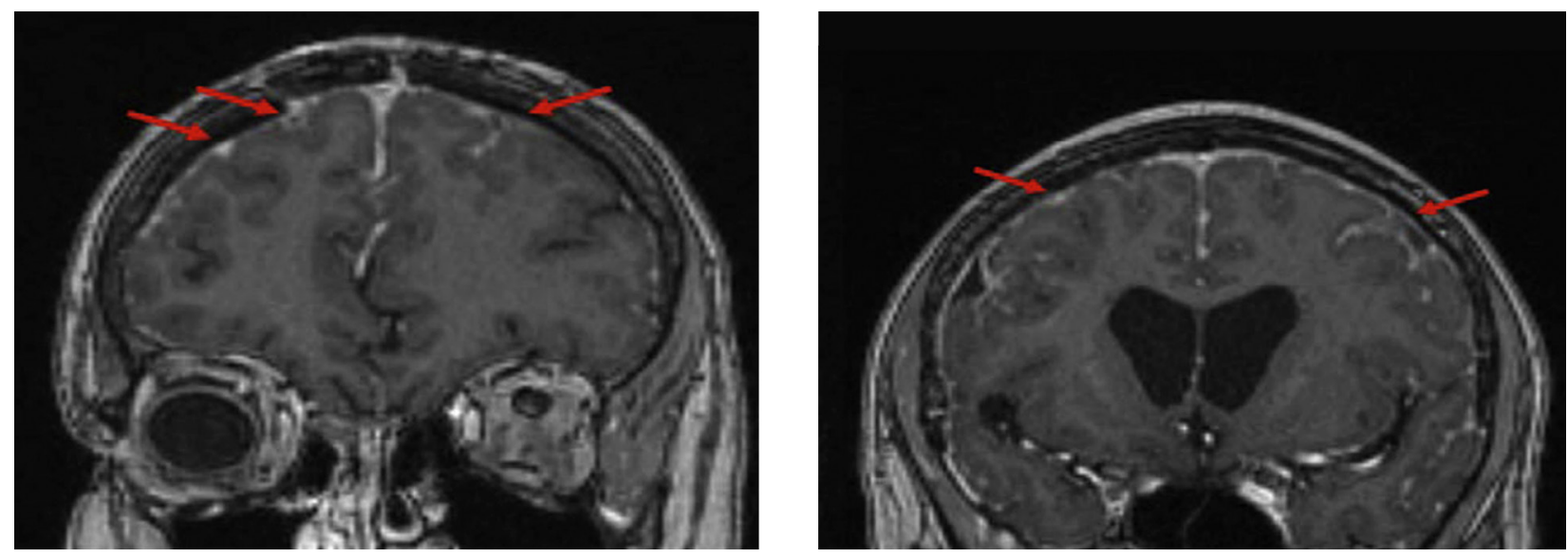

Figure 3. Thirteen Month Follow Up MRI Images (Coronal T1) Demonstrating Regression of The Previous Leptomeningeal Enhancement and Subsequent Progression in New Regions Particularly in The Right Parietal Lobe, With Coinciding Diffusion Restriction

\section{Discussion}

Inflammatory CNS disease with associated leptomeningeal rheumatoid nodules is a rare and unique complication of rheumatoid arthritis. Both pachymeningitis and leptomeningitis may occur. Pachymeningitis, predominantly affecting the dura mater, typically presents with headaches and cranial neuropathies secondary to dural fibrosis or inflammation. If nodular disease primarily in- volves the leptomeninges, then changes in mental status, cognitive deficits, gait disorders, seizures and paresis are more common features (2).

Diagnosis is inherently difficult due to the rarity of the condition and the poor correlation between CNS pathology and peripheral disease activity. Bathon et al. (3) reviewed 19 patients (ten male and nine female) with 
inflammatory CNS disease who were mostly diagnosed on autopsy. Less than half of these patients had active synovitis that was temporally associated with neurological symptoms. The authors concluded that RA duration and activity were not reliable indicators of CNS involvement or disease progression. This conclusion is consistent with our patient's presentation (3).

Definitive diagnosis of leptomeningeal rheumatoid nodules typically requires brain biopsy and confirmation via histopathology. CSF rheumatoid factor is rarely ordered but may be valuable as a strongly positive result can be indicative of disease. CSF findings in rheumatoid meningitis are otherwise typically variable, non-specific and not diagnostic (2). Cerebrospinal fluid analysis often demonstrates a lymphocytic pleocytosis and elevated protein level. Inflammatory cytokines essential in the pathogenesis of rheumatoid arthritis, including TNF- $\alpha$, IL-1 $\beta$ and IL-6, can also be elevated in CSF studies however their significance in unknown (4).

Rheumatoid nodules are the most common pathology identified in rheumatoid meningeal inflammation (63\%) and are histologically identical to subcutaneous rheumatoid nodules. Other changes include non-specific mononuclear inflammatory infiltrates within the leptomeninges or pachymeninges. Less frequently multinucleated giant cells and necrosis are identified (3). Features of vasculitis are identified in $37 \%$ of patients with lymphocyte and plasma cell infiltration of the vessel wall. We hypothesise that the recurrent TIA-like presentations observed in our patient were the result of her leptomeningeal rheumatoid disease and transient focal disruption of cortical circulation.

Few therapies are known to be effective in achieving long-term control or regression of leptomeningeal rheumatoid nodules. Corticosteroids are known to be effective but are often not a sustainable long-term management option (5). Monitoring therapeutic treatment response in the CNS can also be problematic given that patients often lack clinical deficits and their imaging changes generally evolve slowly.

In addition to intermittent short-courses of prednisolone and cessation of methotrexate, our patient was managed with a combination of colchicine at varying doses as well as cessation of methotrexate and leflunomide due to their association with accelerated nodular disease. The patient was switched to tocilizumab, which has been associated with subcutaneous and pulmonary nodule regression in limited case studies $(6,7)$. Since commencing Tocilizumab the patients disease appears to have regressed and stabilised for the past 18 months, although it is difficult to adequately assess the therapeutic benefit given that the natural history of the condition.

Leptomeningeal rheumatoid nodules is a rare condition. The three pertinent points that our patient's case highlights are as follows (1). The differential diagnoses for transient neurological symptoms with MRI evidence of leptomeningeal thickening is large and requires extensive diagnostic workup (2). Meningeal biopsy is crucial to confirm the diagnosis of leptomeningeal rheumatoid nodules and to guide future therapy (3). There is a lack of evidence with respect to satisfactorily treating cerebral rheumatoid nodules and a need for further evidence regarding the efficacy of the newer biological agents.

\section{Authors' Contributions}

Cassie Nesbitt: primary care of patient, writing manuscript, literature review. Luke Wilshire: primary care of patient, assistance with writing manuscript. Doreen Quan: reporting of patients imaging, writing manuscript. Cameron Shaw: primary care of patient, assistance with literature review. Peter Batchelor: primary care of patient, assistance with literature review, critical revision manuscript, supervision.

\section{References}

1. Wallace ZS, Carruthers MN, Khosroshahi A, Carruthers R, Shinagare S, Stemımer-Rachamimov A, et al. IgG4-related disease and hypertrophic pachymeningitis. Medicine (Baltimore). 2013;92(4):206-16.

2. Kupersmith MJ, Martin V, Heller G, Shah A, Mitnick HJ. Idiopathic hypertrophic pachymeningitis. Neurology. 2004;62(5):686-94.

3. Bathon JM, Moreland LW, DiBartolomeo AG. Inflammatory central nervous system involvement in rheumatoid arthritis. Semin Arthritis Rheum.1989;18(4):258-66.

4. Kato T, Hoshi K, Sekijima Y, Matsuda M, Hashimoto T, Otani M, et al. Rheumatoid meningitis: an autopsy report and review of the literature. Clin Rheumatol. 2003;22(6):475-80.

5. Koide R, Isoo A, Ishii K, Uruha A, Bandoh M. Rheumatoid leptomeningitis: rare complication of rheumatoid arthritis. Clin Rheumatol. 2009;28(9):1117-9.

6. Al Attia HM, Abushawish M. Treatment with tocilizumab leads to the disappearance of olecranon rheumatoid nodules. Int J Dermatol. 2012;51(2):197-8

7. Andres M, Vela P, Romera C. Marked improvement of lung rheumatoid nodules after treatment with tocilizumab. Rheumatology (Oxford). 2012;51(6):1132-4. 